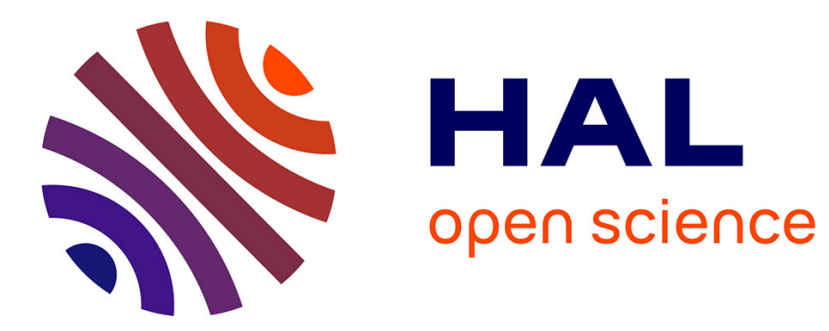

\title{
Sharp error bounds for complex floating-point inversion
}

Claude-Pierre Jeannerod, Nicolas Louvet, Jean-Michel Muller, Antoine Plet

\section{To cite this version:}

Claude-Pierre Jeannerod, Nicolas Louvet, Jean-Michel Muller, Antoine Plet. Sharp error bounds for complex floating-point inversion. Numerical Algorithms, 2016, 73 (3), pp.735-760. 10.1007/s11075016-0115-x . ensl-01195625v2

HAL Id: ensl-01195625

https://hal-ens-lyon.archives-ouvertes.fr/ensl-01195625v2

Submitted on 19 Feb 2016

HAL is a multi-disciplinary open access archive for the deposit and dissemination of scientific research documents, whether they are published or not. The documents may come from teaching and research institutions in France or abroad, or from public or private research centers.
L'archive ouverte pluridisciplinaire HAL, est destinée au dépôt et à la diffusion de documents scientifiques de niveau recherche, publiés ou non, émanant des établissements d'enseignement et de recherche français ou étrangers, des laboratoires publics ou privés. 


\title{
Sharp error bounds for complex floating-point inversion
}

\author{
Claude-Pierre Jeannerod . \\ Nicolas Louvet • Jean-Michel Muller • \\ Antoine Plet
}

the date of receipt and acceptance should be inserted later

\begin{abstract}
We study the accuracy of the classic algorithm for inverting a complex number given by its real and imaginary parts as floating-point numbers. Our analyses are done in binary floating-point arithmetic, with an unbounded exponent range and in precision $p$; we also assume that the basic arithmetic operations $(+,-, \times, /)$ are rounded to nearest, so that the roundoff unit is $u=2^{-p}$. We bound the largest relative error in the computed inverse either in the componentwise or in the normwise sense. We prove the componentwise relative error bound $3 u$ for the complex inversion algorithm (assuming $p \geqslant 4$ ), and we show that this bound is asymptotically optimal (as $p \rightarrow \infty$ ) when $p$ is even, and sharp when using one of the basic IEEE 754 binary formats with an odd precision $(p=53,113)$. This componentwise bound obviously leads to the same bound $3 u$ for the normwise relative error. However, we prove that the smaller bound $2.707131 u$ holds (assuming $p \geqslant 24$ ) for the normwise relative error, and we illustrate the sharpness of this bound for the basic IEEE 754 binary formats $(p=24,53,113)$ using numerical examples.
\end{abstract}

Keywords floating-point arithmetic · rounding error analysis · complex inversion

Claude-Pierre Jeannerod

Inria, Laboratoire LIP (CNRS, ENSL, Inria, UCBL), Université de Lyon, France.

E-mail: claude-pierre.jeannerod@inria.fr

Nicolas Louvet

UCBL, Laboratoire LIP (CNRS, ENSL, Inria, UCBL), Université de Lyon, France.

E-mail: nicolas.louvet@ens-lyon.fr

Jean-Michel Muller

CNRS, Laboratoire LIP (CNRS, ENSL, Inria, UCBL), Université de Lyon, France.

E-mail: jean-michel.muller@ens-lyon.fr

Antoine Plet

ENS de Lyon, Laboratoire LIP (CNRS, ENSL, Inria, UCBL), Université de Lyon, France.

E-mail: antoine.plet@ens-lyon.fr 


\section{Introduction}

This paper deals with the accuracy of the inversion of a nonzero complex number given by its real and imaginary parts as floating-point numbers. We assume that the underlying floating-point arithmetic has radix 2 and precision $p \geqslant 2$, and we also assume an unbounded exponent range, which means that our results apply to practical floating-point calculations according to the IEEE 754 standard [6] as long as underflow and overflow do not occur.

Given a nonzero complex number $a+i b$, its inverse satisfies

$$
z=R+i I, \quad R=\frac{a}{a^{2}+b^{2}}, \quad I=-\frac{b}{a^{2}+b^{2}} .
$$

Assuming $a$ and $b$ are floating-point numbers and denoting by $\mathrm{RN}$ a roundto-nearest function, we focus in this paper on the approximation $\widehat{z}=\widehat{R}+i \widehat{I}$ that can be computed classically in floating-point arithmetic according to

$$
\widehat{R}=\operatorname{RN}\left(\frac{a}{\operatorname{RN}\left(\operatorname{RN}\left(a^{2}\right)+\operatorname{RN}\left(b^{2}\right)\right)}\right)
$$

for the real part, and with a similar expression for the imaginary part $\widehat{I}$. This scheme corresponds to Algorithm 1 below.

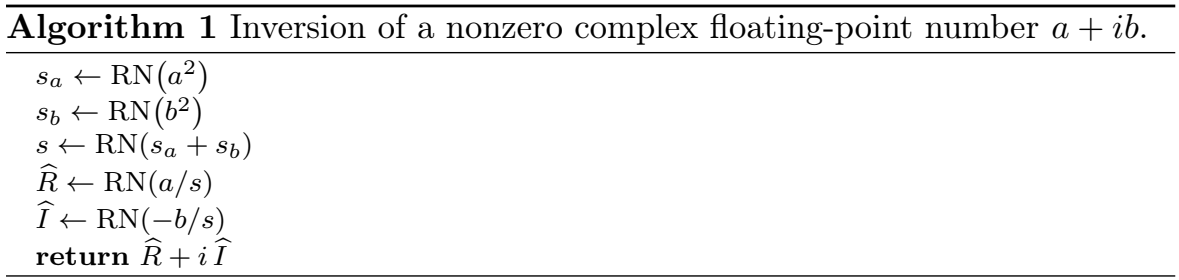

We provide an accuracy analysis of Algorithm 1, for both the componentwise relative error $\mathrm{E}_{\mathrm{C}}=\max (|R-\widehat{R}| /|R|,|I-\widehat{I}| /|I|)$ and the normwise relative error $\mathrm{E}_{\mathrm{N}}=|z-\widehat{z}| /|z|$. In each case, we bound the largest error value by a function $B(p)$ depending only on the precision $p$, and study the tightness of that bound. In this context, we typically distinguish between three levels of quality:

- If we can show that there exist some inputs $a+i b$ parametrized by $p$ and for which the bound is attained for every $p \geqslant p_{0}$ (for a given $p_{0} \geqslant 2$ ), then we say that the bound is optimal.

- If we can show that there exist some inputs parametrized by $p$ and for which the relative error $E(p)$ satisfies $E(p) / B(p) \rightarrow 1$ as $p \rightarrow \infty$, then we say that the bound is asymptotically optimal.

- In some cases, we did not manage to establish (asymptotic) optimality, but have found input examples - either parametrized by $p$, or just for some values of $p$ of practical interest (like those corresponding to the basic IEEE 
754 formats) - for which $E(p)$ is very close to $B(p)$. In this case, we say that the bound is sharp. (See [13] for a similar use of the word "sharp".)

The componentwise relative error generated by Algorithm 1 can easily be bounded as $\mathrm{E}_{\mathrm{C}} \leqslant 3 u+O\left(u^{2}\right)$, where $u=2^{-p}$ is the unit roundoff. Our first contribution is to show that the term $\mathcal{O}\left(u^{2}\right)$ can in fact be removed, which leads to the simpler bound $\mathrm{E}_{\mathrm{C}} \leqslant 3 u$ (assuming $p \geqslant 4$ ). Furthermore, when $p$ is even, we show that this bound is asymptotically optimal by providing floating-point numbers $a$ and $b$ parametrized by $p$ and for which $\mathrm{E}_{\mathrm{C}} \geqslant 3 u-$ $\frac{31}{2} u^{\frac{3}{2}}+\mathcal{O}\left(u^{2}\right)$; when $p$ is odd, we show that the bound $3 u$ is sharp, especially for the corresponding basic IEEE 754 binary formats $(p=53,113)$.

The normwise relative error bound $\mathrm{E}_{\mathrm{N}} \leqslant 3 u+\mathcal{O}\left(u^{2}\right)$ can be found in $[4$, p. 30], and a direct application of our componentwise error analysis leads further to $E_{N} \leqslant 3 u$. The second main contribution of our paper is to show that if $p \geqslant 10$ then the following smaller bound holds: $\mathrm{E}_{\mathrm{N}}<\gamma u+9 u^{2}$, where $\gamma$ is an explicit constant in $(2.70712,2.70713)$. When using for example the IEEE 754 binary32 format $(p=24)$, this implies $\mathrm{E}_{\mathrm{N}}<2.707131 u$. The techniques and the case distinction we use to prove this bound are inspired from [13], but we also extensively use real analysis and differentiation for the treatment of each case. We provide numerical examples to show that the bound we obtain is sharp for the basic IEEE 754 formats $(p=24,53,113)$.

Several authors $[10,11,8,2]$ have suggested ways of avoiding spurious overflows and underflows in complex division, and some of them may be used in Algorithm 1. Of course, if the computation introduces further rounding errors, which is the case for example in Smith's method [10], then our error bounds may not hold anymore. However, following the technique developed by Priest in [8], it is possible to scale $a$ and $b$ by a power of two in order to avoid spurious overflows and underflows without introducing new rounding errors: in that case, our analyses are valid if neither overflow nor underflow occurs during the computation. Nonetheless, we do not deal with scaling techniques here, and focus only on the largest error assuming an unbounded exponent range.

Outline. Section 2 is devoted to the componentwise relative error analysis of Algorithm 1, and Section 3 to its normwise relative error analysis. We conclude in Section 4 with some remarks on the implications of these error analyses for complex floating-point division. The technical parts of the proofs that can be skipped at first reading are gathered in Appendix A.

Assumptions and notation. For any real number $t$, we denote by $\operatorname{RN}(t)$ the binary floating-point number that is nearest to $t$, with a tie-breaking strategy preserving the following properties:

- $\mathrm{RN}\left(2^{k} t\right)=2^{k} \mathrm{RN}(t)$ for any integer $k$;

- $\mathrm{RN}(-t)=-\mathrm{RN}(t)$.

In particular, either the roundTiesToEven or the roundTiesToAway rounding direction attribute defined in the IEEE 754 standard [6] can be used. 
Throughout the paper, we also rely on the following relative error bound [7, p. 232]: for any real number $t$,

$$
\mathrm{RN}(t)=t(1+\epsilon) \quad \text { with } \quad|\epsilon| \leqslant \frac{u}{1+u} .
$$

Note that (3) implies the well-known inequality $|\mathrm{RN}(t)-t| \leqslant u|t|$; see [5, p. 38].

Finally, we use the notation ufp $(t)$ (unit in the first place, introduced in [9]) to denote the weight of the most significant bit of $t$ : if $t \neq 0$ then $\operatorname{ufp}(t)$ is the unique integer power of two such that $1 \leqslant|t| / \mathrm{ufp}(t)<2$, and $\mathrm{ufp}(0)=0$. The usual ulp function (unit in the last place) is related to the ufp function through the relation $\mathrm{ulp}(t)=2 u \cdot \mathrm{ufp}(t)$, so that

$$
|t-\mathrm{RN}(t)| \leqslant \frac{1}{2} \mathrm{ulp}(t)=\mathrm{ufp}(t) u .
$$

\section{Componentwise error bound}

In this section, we focus on the componentwise relative error of Algorithm 1. We note first that since $a+i b$ is nonzero, $R=a /\left(a^{2}+b^{2}\right)$ and $I=-b /\left(a^{2}+b^{2}\right)$ cannot both be zero, and that if one of them is zero then the returned result is very accurate. Assume for example that $R=0$ (the case $I=0$ is similar). In that case, $a=0$ and $I=-1 / b$. Using the bound in (3), it is then easily checked that the values $\widehat{R}$ and $\widehat{I}$ returned by the algorithm are as follows:

- $\widehat{R}=0$, which means that the real part is computed exactly;

- $\widehat{I}=-\mathrm{RN}\left(b / \mathrm{RN}\left(b^{2}\right)\right)$ and the relative error on the imaginary part is bounded by $2 u$ (and thus smaller than the bound we are going to give in the general case).

Therefore, the rest of this section is devoted to analyzing $\mathrm{E}_{\mathrm{C}}=\max (\mid R-$ $\widehat{R}|/| R|| I-,\widehat{I}|/| I \mid)$ for $R$ and $I$ nonzero. Repeated applications of the bound in (3) give immediately $\mathrm{E}_{\mathrm{C}} \leqslant 3 u+\mathcal{O}\left(u^{2}\right)$. We show below that if $p \geqslant 4$ then the $\mathcal{O}\left(u^{2}\right)$ term can in fact be removed, leading to the simpler bound $3 u$.

To do this, we prove that if $p \neq 3$ then the relative error bound $u /(1+u)$ in (3) can be replaced by $u /(1+3 u)$ when evaluating a square $\operatorname{RN}\left(a^{2}\right)$ instead of a general product. (When $p=3$, it is easily checked that the bound $u /(1+u)$ is attained when squaring the floating-point numbers $3 / 2 \cdot 2^{e}, e \in \mathbb{Z}$.) This slight refinement will turn out to be enough to show that Algorithm 1 satisfies $\mathrm{E}_{\mathrm{C}} \leqslant 3 u$.

Lemma 1 Let a be a floating-point number. If $p \neq 3$ then $\left|a^{2}-(2+2 u)\right| \geqslant 4 u^{2}$.

Proof If $|a|<1$ then $\left|a^{2}-(2+2 u)\right|>1+2 u$, and the result follows from the fact that $1+2 u>4 u^{2}$ when $p>0$. Assume now that $|a| \geqslant 1$. To handle this case, we show first that

$$
a^{2}=2+2 u \quad \Rightarrow \quad p=3 .
$$


Since $|a|$ is a floating-point number not smaller than 1 , there exists a positive integer $A$ such that $|a|=A \cdot 2^{1-p}=A \cdot 2 u$. The equality $a^{2}=2+2 u$ is thus equivalent to $A^{2}=\left(2^{p}+1\right) \cdot 2^{p-1}$ and, using the (unique) decomposition $A=(2 B+1) \cdot 2^{C}$ with $B, C \in \mathbb{N}$, it can also be rewritten $(2 B+1)^{2} \cdot 2^{2 C}=$ $\left(2^{p}+1\right) \cdot 2^{p-1}$. Now, $p>0$ implies that $2^{p}+1$ is odd and at least 3 , so $B \neq 0$ and $(2 B+1)^{2}=2^{p}+1$. The latter equality can be rewritten as $4 B(B+1)=2^{p}$ and its unique solution over $\mathbb{N}_{>0}^{2}$ is $(B, p)=(1,3)$, so (5) follows.

If $p \neq 3$ then, by (5) we have $a^{2} \neq 2+2 u$, that is, $A^{2} \neq\left(2^{p}+1\right) \cdot 2^{p-1}$. Since the latter inequality involves only integers, it is equivalent to $\mid A^{2}-\left(2^{p}+\right.$ 1) $\cdot 2^{p-1} \mid \geqslant 1$ and thus to $\left|a^{2}-(2+2 u)\right| \geqslant 4 u^{2}$.

Lemma 2 Let a be a floating-point number. If $p \neq 3$ then $\operatorname{RN}\left(a^{2}\right)=a^{2}(1+\epsilon)$ with $|\epsilon| \leqslant u /(1+3 u)$.

Proof We can assume that $1 \leqslant a<2$. If $a=1$ then $\operatorname{RN}\left(a^{2}\right)=a^{2}$ and the result is clear. If $1<a<\sqrt{2}$ then it follows from $a$ being a floating-point number that $p \geqslant 4$ and that $a$ belongs to the non-empty interval $[1+2 u, \sqrt{2})$. Consequently, $1+4 u<a^{2}<2$ and thus $|\epsilon| \leqslant u$ ufp $\left(a^{2}\right) / a^{2}=u / a^{2}<u /(1+4 u)$. Finally, if $\sqrt{2}<a<2$ then $2<a^{2}<4$ and, by Lemma 1 , it suffices to consider the following four subcases:

- If $2<a^{2} \leqslant 2+2 u-4 u^{2}$ then $\operatorname{RN}\left(a^{2}\right)=2$ and, therefore,

$$
|\epsilon|=1-\frac{2}{a^{2}} \leqslant 1-\frac{2}{2+2 u-4 u^{2}} \leqslant \frac{u}{1+3 u} .
$$

- If $2+2 u+4 u^{2} \leqslant a^{2}<2+4 u$ then $\operatorname{RN}\left(a^{2}\right)=2+4 u$ and, therefore,

$$
|\epsilon|=\frac{2+4 u}{a^{2}}-1 \leqslant \frac{2+4 u}{2+2 u+4 u^{2}}-1 \leqslant \frac{u}{1+3 u} .
$$

- If $2+4 u \leqslant a^{2}<2+6 u$ then $\operatorname{RN}\left(a^{2}\right)=2+4 u$ and, therefore,

$$
|\epsilon|=1-\frac{2+4 u}{a^{2}} \leqslant 1-\frac{2+4 u}{2+6 u}=\frac{u}{1+3 u} .
$$

- If $2+6 u \leqslant a^{2}<4$ then $\operatorname{ufp}\left(a^{2}\right)=2$ and $|\epsilon| \leqslant 2 u / a^{2} \leqslant 2 u /(2+6 u)=$ $u /(1+3 u)$.

Theorem 1 If $p \geqslant 4$ then the componentwise relative error for Algorithm 1 satisfies $\mathrm{E}_{\mathrm{C}} \leqslant 3 u$.

Proof Due to the symmetry of Algorithm 1, it suffices to show that $|R-\widehat{R}| \leqslant$ $3 u|R|$. From (3) and Lemma 2 we have

$s_{a}=a^{2}\left(1+\epsilon_{a}\right), \quad s_{b}=b^{2}\left(1+\epsilon_{b}\right), \quad s=\left(s_{a}+s_{b}\right)\left(1+\epsilon_{s}\right), \quad \widehat{R}=\frac{a}{s}\left(1+\epsilon_{R}\right)$

with $\left|\epsilon_{a}\right|,\left|\epsilon_{b}\right| \leqslant u /(1+3 u)$ and $\left|\epsilon_{s}\right|,\left|\epsilon_{R}\right| \leqslant u /(1+u)$. Hence

$$
\widehat{R}=\frac{a}{a^{2}\left(1+\epsilon_{a}\right)+b^{2}\left(1+\epsilon_{b}\right)} \cdot \frac{1+\epsilon_{R}}{1+\epsilon_{s}}
$$


and, using $R=a /\left(a^{2}+b^{2}\right)$, we deduce that $\varphi R \leqslant \widehat{R} \leqslant \varphi^{\prime} R$ with

$$
\varphi:=\frac{1-\frac{u}{1+u}}{\left(1+\frac{u}{1+3 u}\right)\left(1+\frac{u}{1+u}\right)} \quad \text { and } \quad \varphi^{\prime}:=\frac{1+\frac{u}{1+u}}{\left(1-\frac{u}{1+3 u}\right)\left(1-\frac{u}{1+u}\right)} .
$$

It is easily checked that $\varphi>1-3 u$ and $\varphi^{\prime}=1+3 u$, which completes the proof.

We conclude this section by showing that the componentwise bound $\mathrm{E}_{\mathrm{C}} \leqslant$ $3 u$ is sharp. More precisely, when the precision $p$ is even, the following example shows that the componentwise error bound $3 u$ is asymptotically optimal as $p \rightarrow \infty$. Assuming an even $p \geqslant 12$, let us consider the following binary floatingpoint numbers in precision $p$ :

$$
\begin{aligned}
& a=2^{\frac{p}{2}-1}+5 \cdot 2^{-2}+2^{-\frac{p}{2}+2}, \\
& b=2^{p-1}+2^{\frac{p}{2}-1}+1 .
\end{aligned}
$$

With these values as inputs of Algorithm 1, we have (the details are provided in Appendix A.1):

$$
\begin{aligned}
s_{a} & =2^{p-2}+5 \cdot 2^{\frac{p}{2}-2}+11 \cdot 2^{-1}, \\
s_{b} & =2^{2 p-2}+2^{\frac{3 p}{2}-1}+3 \cdot 2^{p-1}, \\
s & =2^{2 p-2}+2^{\frac{3 p}{2}-1}+2^{p+1} .
\end{aligned}
$$

From this we deduce

$$
\frac{a}{s}=2^{-\frac{3 p}{2}+1}+2^{-2 p}-2^{-\frac{5 p}{2}+1}-2^{-3 p+2}+\mathcal{O}\left(2^{-\frac{7 p}{2}}\right),
$$

and ulp $\left(\frac{a}{s}\right)=2^{-\frac{5 p}{2}+2}$. Then, defining the floating-point number $\tau$ by

$$
\tau=2^{-\frac{3 p}{2}+1}+2^{-2 p}-2^{-\frac{5 p}{2}+2},
$$

it can be checked that

$$
\left|\frac{a}{s}-\tau\right|=\frac{2^{-\frac{5 p}{2}+1}+2^{-\frac{7 p}{2}+5}}{1+2^{-\frac{p}{2}+1}+2^{-p+3}}<\frac{1}{2} \operatorname{ulp}\left(\frac{a}{s}\right) .
$$

Hence $\widehat{R}=\mathrm{RN}\left(\frac{a}{s}\right)=\tau$, which together with $R=a /\left(a^{2}+b^{2}\right)$ leads to

$$
\frac{R-\widehat{R}}{R}=3 u-\frac{31}{2} u^{\frac{3}{2}}+\mathcal{O}\left(u^{2}\right) .
$$

As a consequence, in this example the componentwise relative error in the computed $\widehat{z}$ is at least $3 u-\frac{31}{2} u^{\frac{3}{2}}+\mathcal{O}\left(u^{2}\right)$, which shows the asymptotic optimality (as $p \rightarrow \infty$ ) of the bound when $p$ is even.

When $p$ is odd, we have not found an input set parametrized by the precision to prove the asymptotic optimality of the error bound $3 u$. However, we illustrate the sharpness of the bound by numerical examples in Table 1. 


\begin{tabular}{|l|l|l|}
\hline$p$ & Inputs $a$ and $b$ & $E_{C} / u$ \\
\hline 15 & $\begin{array}{l}a=16732 \\
b=23252 \cdot 2^{3}\end{array}$ & $2.93047 \ldots$ \\
\hline 17 & $\begin{array}{l}a=66078 \\
b=93014 \cdot 2^{8}\end{array}$ & $2.96359 \ldots$ \\
\hline 19 & $\begin{array}{l}a=131435 \\
b=370969 \cdot 2^{8}\end{array}$ & $2.98509 \ldots$ \\
\hline 53 & $\begin{array}{l}a=4508053433127332 \\
b=6369149602646415 \cdot 2^{16}\end{array}$ & $2.97894 \ldots$ \\
\hline 113 & $\begin{array}{l}a=5192393427440123027423416459819356 \\
b=7343016638055329519853569740503421 \cdot 2^{16}\end{array}$ & $2.97647 \ldots$ \\
\hline
\end{tabular}

Table 1 Examples with $p$ odd and a componentwise relative error close to $3 u$.

\section{Normwise error bound}

In this section, we are interested in the normwise relative error of Algorithm 1, that is,

$$
\mathrm{E}_{\mathrm{N}}=\sqrt{a^{2}+b^{2}} \sqrt{(R-\widehat{R})^{2}+(I-\widehat{I})^{2}} .
$$

The analysis is done in radix 2 and precision $p$, and we assume that overflows and underflows never occur. If we apply directly the componentwise bound obtained in Section 2, we end up with the normwise error bound $\mathrm{E}_{\mathrm{N}} \leqslant 3 u$. In this section, we establish the following result, which achieves a smaller bound by keeping track of the correlations between the various rounding errors committed by the algorithm.

Theorem 2 If $p \geqslant 10$ then the normwise relative error for Algorithm 1 satisfies $\mathrm{E}_{\mathrm{N}} \leqslant \gamma u+9 u^{2}$, where $\gamma$ is defined by

$$
\gamma=\frac{\sqrt{8778980525057+16793600(8 \sqrt{2}-\sqrt{127})-550842155008 \sqrt{254}}}{8192(16-\sqrt{254})},
$$

and is such that $\gamma \in(2.70712,2.70713)$.

If $p \geqslant 10, \mathrm{E}_{\mathrm{N}}<2.70713 u+9 u^{2}$ is therefore a rigorous bound for the normwise error of Algorithm 1. It should also be noticed that the second order term in the error bound can be absorbed by the first order term, at the cost of a slight overestimation: for example, for $p \geqslant 24$, we have $9 u=9 \cdot 2^{-24}<$ $10^{-6}$ so that $\mathrm{E}_{\mathrm{N}}<2.707131 u$. The numerical examples listed in Table 2 show that the error bound of Theorem 2 is sharp for the basic IEEE 754 formats $(p=24,53,113)$.

\subsection{Preliminaries}

The first step in the error analysis of Algorithm 1 is to reduce the input domain. Since the function RN is symmetric with respect to zero, the signs of 


\begin{tabular}{|l|l|l|}
\hline$p$ & Inputs $a$ and $b$ & $E_{N} / u$ \\
\hline 24 & $\begin{array}{l}a=11863283 \\
b=11865457 \cdot 2^{12}\end{array}$ & $2.69090 \ldots$ \\
\hline 53 & $\begin{array}{l}a=4503599709991314 \\
b=6369051770002436 \cdot 2^{26}\end{array}$ & $2.70679 \ldots$ \\
\hline 113 & $\begin{array}{l}a=2^{112} \\
b=7343016637207171132572330391109909 \cdot 2^{56}\end{array}$ & $2.70559 \ldots$ \\
\hline
\end{tabular}

Table 2 Examples with a normwise relative error close to $\gamma u$.

$a$ and $b$ are not relevant and we can assume that both $a$ and $b$ are nonnegative. Swapping the inputs $a$ and $b$ does not affect the relative error; moreover, if $a=0$, then a simple analysis, based on (3), leads to the upper bound $2 u$ for $\mathrm{E}_{\mathrm{N}}$, so we can assume that $0<a \leqslant b$. Finally, multiplying or dividing by two both $a$ and $b$ does not affect either the relative error, and we can restrict the analysis to the case $1 \leqslant b<2$.

From the definition of the ufp function and this input range reduction, we know that $u f p\left(b^{2}\right) \in\{1,2\}$. Moreover, $b$ is a floating-point number, so that $1 \leqslant b \leqslant 2-2 u$ and thus $1 \leqslant b^{2} \leqslant 4-4 u$. Since $4-4 u$ is a floating-point number, and using the monotonicity of the rounding function $\mathrm{RN}$, we deduce that $1 \leqslant s_{b}<4$. Using again the monotonicity of RN, we also deduce that $0<s_{a}<4$. Hence $1<s_{a}+s_{b}<8$, which implies ufp $\left(s_{a}+s_{b}\right) \in\{1,2,4\}$.

We now define $\delta_{a}, \delta_{b}, \delta_{s}, \delta_{R}$, and $\delta_{I}$ as follows:

$$
\begin{aligned}
s_{a} & =a^{2}+\delta_{a} u, & & \left|\delta_{a}\right| \leqslant \operatorname{ufp}\left(a^{2}\right), \\
s_{b} & =b^{2}+\delta_{b} u, & & \left|\delta_{b}\right| \leqslant \operatorname{ufp}\left(b^{2}\right), \\
s & =s_{a}+s_{b}+\delta_{s} u, & & \left|\delta_{s}\right| \leqslant \operatorname{ufp}\left(s_{a}+s_{b}\right), \\
\widehat{R} & =\frac{a}{s}+\delta_{R} u, & & \left|\delta_{R}\right| \leqslant \operatorname{ufp}\left(\frac{a}{s}\right), \\
\widehat{I} & =-\left(\frac{b}{s}+\delta_{I} u\right), & & \left|\delta_{I}\right| \leqslant \operatorname{ufp}\left(\frac{b}{s}\right) .
\end{aligned}
$$

Let us also define $\delta=\delta_{a}+\delta_{b}+\delta_{s}$ and $\epsilon=\frac{|\delta|}{a^{2}+b^{2}}$, so that $|\delta| u$ and $\epsilon u$ are the absolute and relative errors, respectively, in the evaluation of $a^{2}+b^{2}$. Since $0<a \leqslant b, \operatorname{ufp}\left(b^{2}\right) \leqslant 2$ and $\operatorname{ufp}\left(s_{a}+s_{b}\right) \leqslant 4$, we deduce that $|\delta| \leqslant 8$. Moreover, it can be deduced from (3) that $\epsilon \leqslant 2$. (This bound on $\epsilon$ already appeared in $[3$, p. 1471].)

With these notations, we have

$$
R-\widehat{R}=\frac{a}{s\left(a^{2}+b^{2}\right)} \delta u-\delta_{R} u
$$

and since a similar expression holds for $I-\widehat{I}$, we arrive at

$$
\frac{\mathrm{E}_{\mathrm{N}}^{2}}{u^{2}}=\left(a^{2}+b^{2}\right)\left(\delta_{R}^{2}+\delta_{I}^{2}\right)-2 \frac{\delta\left(a \delta_{R}+b \delta_{I}\right)}{a^{2}+b^{2}+\delta u}+\left(\frac{\delta}{a^{2}+b^{2}+\delta u}\right)^{2}
$$


Then, using the triangular inequality, we obtain

$$
\begin{aligned}
\frac{\mathrm{E}_{\mathrm{N}}^{2}}{u^{2}} \leqslant\left(a^{2}+b^{2}\right)\left(\operatorname{ufp}\left(\frac{a}{s}\right)^{2}\right. & \left.+\operatorname{ufp}\left(\frac{b}{s}\right)^{2}\right) \\
& +2 \frac{|\delta|\left(\operatorname{ufp}\left(\frac{a}{s}\right) a+\operatorname{ufp}\left(\frac{b}{s}\right) b\right)}{a^{2}+b^{2}-|\delta| u}+\left(\frac{\delta}{a^{2}+b^{2}-|\delta| u}\right)^{2} .
\end{aligned}
$$

For $p \geqslant 2, \epsilon u<1$ and we use the equality $\frac{1}{a^{2}+b^{2}-|\delta| u}=\frac{1}{a^{2}+b^{2}}\left(1+\frac{\epsilon}{1-\epsilon u} u\right)$ and the inequality $\left(1+\frac{\epsilon}{1-\epsilon u} u\right)^{2} \leqslant 1+\frac{2 \epsilon}{(1-\epsilon u)^{2}} u$ to get

$$
\mathrm{E}_{\mathrm{N}}^{2} \leqslant f_{2}(a, b) u^{2}+f_{3}(a, b) u^{3}
$$

with

$$
\begin{aligned}
f_{2}(a, b)=\left(a^{2}+b^{2}\right)\left(\operatorname{ufp}\left(\frac{a}{s}\right)^{2}\right. & \left.+\operatorname{ufp}\left(\frac{b}{s}\right)^{2}\right) \\
& +2 \frac{|\delta|\left(\operatorname{ufp}\left(\frac{a}{s}\right) a+\operatorname{ufp}\left(\frac{b}{s}\right) b\right)}{a^{2}+b^{2}}+\left(\frac{\delta}{a^{2}+b^{2}}\right)^{2}
\end{aligned}
$$

and

$$
f_{3}(a, b)=2\left(\operatorname{ufp}\left(\frac{a}{s}\right) a+\operatorname{ufp}\left(\frac{b}{s}\right) b\right) \frac{\epsilon^{2}}{1-\epsilon u}+\frac{2 \epsilon^{3}}{(1-\epsilon u)^{2}} .
$$

From (4), we have

$$
\operatorname{ufp}\left(\frac{a}{s}\right) a+\operatorname{ufp}\left(\frac{b}{s}\right) b \leqslant \frac{a^{2}+b^{2}}{s} \leqslant \frac{a^{2}+b^{2}}{a^{2}+b^{2}-|\delta| u}=\frac{1}{1-\epsilon u},
$$

and since $0 \leqslant \epsilon \leqslant 2$, it follows that $f_{3}(a, b) \leqslant \frac{2 \epsilon^{2}(1+\epsilon)}{(1-\epsilon u)^{2}}<25$ for $p \geqslant 10$. Moreover, if $f_{2}$ is upper bounded by $\kappa$, we can conclude from (7) that

$$
\mathrm{E}_{\mathrm{N}} \leqslant \sqrt{\kappa} u+\frac{25}{2 \sqrt{\kappa}} u^{2}
$$

\subsection{Taking care of some corner cases}

We can first roughly bound $f_{2}$ using the inequality ufp $(t) \leqslant|t|$, valid for any real $t$, which will allow us to conclude in some particular cases and to further reduce the input domain. From (8) we have

$$
\begin{aligned}
f_{2}(a, b) & \leqslant\left(\frac{a^{2}+b^{2}}{a^{2}+b^{2}-|\delta| u}\right)^{2}+2 \frac{|\delta|\left(a^{2}+b^{2}\right)}{\left(a^{2}+b^{2}\right)\left(a^{2}+b^{2}-|\delta| u\right)}+\left(\frac{\delta}{a^{2}+b^{2}}\right)^{2} \\
& =\left(1+\epsilon+\frac{\epsilon}{1-\epsilon u} u\right)^{2}
\end{aligned}
$$


This last bound is increasing with respect to $\epsilon$ and $u$ (i.e., decreasing with respect to the precision $p$ ). Therefore, if $\epsilon \leqslant 1+\frac{\sqrt{2}}{2}+u$, and as soon as $p \geqslant 5$, we have $f_{2}(a, b) \leqslant\left(2+\frac{\sqrt{2}}{2}+3 u\right)^{2}$ and, from $(9)$,

$$
\mathrm{E}_{\mathrm{N}} \leqslant\left(2+\frac{\sqrt{2}}{2}\right) u+8 u^{2}
$$

Below are five cases that lead to the inequality $\epsilon \leqslant 1+\frac{\sqrt{2}}{2}+u$, so they can be ignored in the following analysis.

- If $a=b$, then $s_{a}=s_{b}$ and $s=s_{a}+s_{b}$ so that $\delta_{s}=0$ and one can check that $\epsilon \leqslant 1$. In this case, the previous bound (10) holds and we can continue the analysis assuming that

$$
a<b \text {. }
$$

- If $b=1$, then $s_{b}=b^{2}=1$ and $\delta_{b}=0$. Moreover, from (11) we have $a<1$, so that $s_{a}<1$, which implies ufp $\left(1+s_{a}\right)=1$ and $\epsilon \leqslant 1$. Again, the bound (10) holds and we can continue the analysis assuming that $1<b$. In fact, since $b$ is a floating-point number, we can assume that

$$
1+2 u \leqslant b .
$$

- If $a=1$, then $\delta_{a}=0$ and we can distinguish three cases. If $u f p\left(b^{2}\right)=1$ then $\mathrm{ufp}\left(1+s_{b}\right)=2$ and $\epsilon \leqslant \frac{3}{2}$. If $\operatorname{ufp}\left(b^{2}\right)=2$ then either ufp $\left(1+s_{b}\right)=2$ which implies $\epsilon \leqslant \frac{4}{3}$, or $u f p\left(1+s_{b}\right)=4$ and then $\epsilon \leqslant \frac{3}{2}+u$. In all these cases, (10) holds, hence we can assume now that

$$
a \neq 1 \text {. }
$$

- If $a^{2}+b^{2}<\operatorname{ufp}\left(s_{a}+s_{b}\right)$, then we have $\left(s_{a}+s_{b}\right)-\operatorname{ufp}\left(s_{a}+s_{b}\right)<\left(\delta_{a}+\delta_{b}\right) u \leqslant$ $\left(a^{2}+b^{2}\right) u<\operatorname{ufp}\left(s_{a}+s_{b}\right) u=\frac{1}{2} \operatorname{ulp}\left(s_{a}+s_{b}\right)$. Since ufp $\left(s_{a}+s_{b}\right)$ is a floatingpoint number, we can deduce that $s=\operatorname{RN}\left(s_{a}+s_{b}\right)=\operatorname{ufp}\left(s_{a}+s_{b}\right)$ hence $\epsilon \leqslant 1$ and (10) holds. In the following, we can then assume that

$$
\operatorname{ufp}\left(s_{a}+s_{b}\right) \leqslant a^{2}+b^{2} .
$$

- One last case is when $s_{a}+s_{b} \geqslant \sqrt{2} \mathrm{ufp}\left(s_{a}+s_{b}\right)$. In this case, $\epsilon \leqslant 1+\frac{\sqrt{2}}{2}+u$ and the previous bound (10) holds. Therefore, we now assume that

$$
s_{a}+s_{b}<\sqrt{2} \mathrm{ufp}\left(s_{a}+s_{b}\right) .
$$

\subsection{Overview of the case analysis}

The analysis goes through the possible values of $\operatorname{ufp}\left(s_{a}+s_{b}\right)$, which are 1,2 , and 4 . In each case, we first deduce upper bounds for $\operatorname{ufp}\left(b^{2}\right), \operatorname{ufp}\left(\frac{a}{s}\right)$, and ufp $\left(\frac{b}{s}\right)$. This leads to a new function $g$, which is greater than or equal to $f_{2}$, and which depends on $a$ and $b$ as well as on a third parameter, $e$, defined as the unique integer such that

$$
\operatorname{ufp}\left(a^{2}\right)=2^{-e}
$$


The function $g$ does not involve floating-point operations anymore and can be seen as a continuous and differentiable function over real inputs. We then look for an upper bound on this function over a restricted domain $D$ containing all the floating-point numbers we are interested in. For this latter step, we mainly use real analysis, especially partial derivatives. In some cases, we can maximize with respect to $a$ and $b$ at the same time. The last step is always to maximize with respect to $e$, using the change of variable $x=2^{-e}$ and considering $x$ as a continuous variable.

The analysis is split into seven cases depending on the values of some ufp functions involved in the definition (8) of $f_{2}$. Note that, since $a^{2}<4$, we have $e \geqslant-1$. In each case but the last one, we end up with a bound smaller than or equal to $\left(2+\frac{\sqrt{2}}{2}\right)^{2}$ for $f_{2}$, from which we conclude using (9) that $\mathrm{E}_{\mathrm{N}} \leqslant\left(2+\frac{\sqrt{2}}{2}\right) u+5 u^{2}$. The last case is similar although we have a slightly larger bound $\gamma^{2}+20 u$ for $f_{2}$ (we have $2+\frac{\sqrt{2}}{2}=2.70710 \ldots$, while $\gamma=2.70712 \ldots)$, which leads to $\mathrm{E}_{\mathrm{N}} \leqslant \gamma u+9 u^{2}$. The table below summarizes the bounds in each case, under the assumptions (11) to (15).

\begin{tabular}{|c|c|c|c|c|c|}
\hline $\mathrm{ufp}\left(s_{a}+s_{b}\right)$ & $\mathrm{ufp}\left(b^{2}\right)$ & $e$ & $\mathrm{ufp}\left(\frac{a}{s}\right)$ & $f_{2}$ & $\mathrm{E}_{\mathrm{N}}$ \\
\hline 1 & 1 & $\geqslant 2$ & $\leqslant 2^{-\frac{e}{2}}$ & 6.565 & $2.6 u$ \\
\hline \multirow{2}{*}{4} & 2 & $=-1$ & $\leqslant \frac{1}{4}$ & $\left(2+\frac{\sqrt{2}}{2}\right)^{2}$ & $\left(2+\frac{\sqrt{2}}{2}\right) u+5 u^{2}$ \\
\cline { 3 - 6 } & \multirow{2}{*}{2} & $\geqslant 0$ & $\leqslant 2^{-2-\frac{e}{2}}$ & $\left(\frac{7}{4}+\frac{\sqrt{2}}{2}\right)^{2}$ & $2.5 u$ \\
\hline \multirow{3}{*}{2} & \multirow{2}{*}{1} & $=0$ & $\leqslant \frac{1}{4}$ & $\left(\frac{5}{2}\right)^{2}$ & $\frac{5}{2} u+5 u^{2}$ \\
\cline { 3 - 6 } & \multirow{2}{*}{2} & $\geqslant 1$ & $\leqslant 2^{-\frac{3+e}{2}}$ & $\left(2+\frac{\sqrt{2}}{2}\right)^{2}$ & $\left(2+\frac{\sqrt{2}}{2}\right) u+5 u^{2}$ \\
\cline { 2 - 6 } & & $\geqslant 2$, even & $=2^{-1-\frac{e}{2}}$ & $\gamma^{2}+20 u$ & $\gamma u+9 u^{2}$ \\
\hline
\end{tabular}

We give all the details of the analysis of the first case. For the other cases, we only give a sketch of the analysis, while deferring the details to Appendices A.2 to A.7.

\subsection{Case ufp $\left(s_{a}+s_{b}\right)=1$}

In this case, we can deduce from $(15)$ that $1 \leqslant s_{a}+s_{b}<\sqrt{2}$. As a consequence, we must have $b<\sqrt{2}$ (otherwise we would have $s_{a}+s_{b}>2$ ), hence

$$
\operatorname{ufp}\left(b^{2}\right)=1
$$

Since $s_{a}<\sqrt{2}-1<\frac{1}{2}$ and $s_{a}=\mathrm{RN}\left(a^{2}\right)$, we have $a^{2}<\frac{1}{2}$, and

$$
e \geqslant 2
$$


Moreover, we know from (12) that $b \geqslant 1+2 u$ so we have $b^{2} \geqslant b(1+2 u) \geqslant b+2 u$, which is a floating-point number because ufp $(b)=1$. Consequently $s_{b} \geqslant b+2 u$ and $s \geqslant s_{a}+s_{b}-u \geqslant s_{a}+b+u>b$, hence $\frac{b}{s}<1$, which implies

$$
\operatorname{ufp}\left(\frac{b}{s}\right) \leqslant \frac{1}{2}
$$

Finally, $s=\mathrm{RN}\left(s_{a}+s_{b}\right) \geqslant 1$ so $\frac{a}{s} \leqslant a<2^{\frac{1-e}{2}}$ and

$$
\operatorname{ufp}\left(\frac{a}{s}\right) \leqslant 2^{-\frac{e}{2}} .
$$

Therefore, using (8) we deduce in this case that $f_{2}(a, b) \leqslant g_{1}(a, b, e)$, with

$$
g_{1}(a, b, e):=\left(a^{2}+b^{2}\right)\left(2^{-e}+\frac{1}{4}\right)+2 \frac{\left(2+2^{-e}\right)\left(2^{-\frac{e}{2}} a+\frac{b}{2}\right)}{a^{2}+b^{2}}+\left(\frac{2+2^{-e}}{a^{2}+b^{2}}\right)^{2} .
$$

Let us now characterize explicitly the domain over which we will bound $g_{1}(a, b, e)$. First, we know that $2^{-\frac{e}{2}} \leqslant a<2^{\frac{1-e}{2}}$. Next, since $s_{a}+s_{b}<\sqrt{2}$ and $s_{a}>0$, we have $s_{b}<\sqrt{2}$, so that $b^{2}<\sqrt{2}+u$ and $1<b<\sqrt{\sqrt{2}+u}$. Finally, we have $a^{2}+b^{2} \leqslant s_{a}+\operatorname{ufp}\left(a^{2}\right) u+s_{b}+\operatorname{ufp}\left(b^{2}\right) u<\sqrt{2}+\frac{5}{4} u$, which concludes the domain analysis: it suffices to look for an upper bound for $g_{1}$ over the domain

$D_{1}:=\left\{(a, b, e) \mid 2^{-\frac{e}{2}} \leqslant a<2^{\frac{1-e}{2}}, 1 \leqslant b<\sqrt{\sqrt{2}+u}, a^{2}+b^{2}<\sqrt{2}+\frac{5}{4} u, e \geqslant 2\right\}$.

We now compute the partial derivatives of $g_{1}$ with respect to $a$ and $b$,

$$
\begin{aligned}
& \frac{\partial g_{1}}{\partial a}=2 a\left(2^{-e}+\frac{1}{4}\right)+\frac{2+2^{-e}}{a^{2}+b^{2}} 2^{1-\frac{e}{2}}-4 a \frac{\left(2+2^{-e}\right)\left(2^{-\frac{e}{2}} a+\frac{b}{2}\right)}{\left(a^{2}+b^{2}\right)^{2}}-4 a \frac{\left(2+2^{-e}\right)^{2}}{\left(a^{2}+b^{2}\right)^{3}}, \\
& \frac{\partial g_{1}}{\partial b}=2 b\left(2^{-e}+\frac{1}{4}\right)+\frac{2+2^{-e}}{a^{2}+b^{2}}-4 b \frac{\left(2+2^{-e}\right)\left(2^{-\frac{e}{2}} a+\frac{b}{2}\right)}{\left(a^{2}+b^{2}\right)^{2}}-4 b \frac{\left(2+2^{-e}\right)^{2}}{\left(a^{2}+b^{2}\right)^{3}},
\end{aligned}
$$

and the next step is to prove that they are both negative over the domain $D_{1}$. Since $\frac{1}{b} \frac{\partial}{\partial b} g_{1}(a, b, e)-\frac{1}{a} \frac{\partial}{\partial a} g_{1}(a, b, e)=\frac{2+2^{-e}}{a^{2}+b^{2}}\left(\frac{1}{b}-\frac{1}{a} 2^{1-\frac{e}{2}}\right)<0$ over $D_{1}$, it is sufficient to prove that $\frac{\partial}{\partial a} g_{1}(a, b, e)<0$. Since $2 a \frac{2+2^{-e}}{a^{2}+b^{2}}>0$, we can rewrite this inequality as

$$
\frac{\left(2^{-e}+\frac{1}{4}\right)\left(a^{2}+b^{2}\right)}{2+2^{-e}}+\frac{2^{-\frac{e}{2}}}{a}<2 \frac{2^{-\frac{e}{2}} a+\frac{b}{2}}{a^{2}+b^{2}}+2 \frac{2+2^{-e}}{\left(a^{2}+b^{2}\right)^{2}} .
$$

This inequality follows from the following three relations:

$$
\begin{aligned}
& \frac{\left(2^{-e}+\frac{1}{4}\right)\left(a^{2}+b^{2}\right)}{2+2^{-e}}+\frac{2^{-\frac{e}{2}}}{a}<\frac{\sqrt{2}+\frac{5}{4} u}{4}+1 \quad \text { for }(a, b, e) \in D_{1}, \\
& \frac{\sqrt{2}+\frac{5}{4} u}{4}+1<\frac{1}{\sqrt{2}+\frac{5}{4} u}+\frac{4}{\left(\sqrt{2}+\frac{5}{4} u\right)^{2}} \quad \text { for } p \geqslant 3, \\
& \frac{1}{\sqrt{2}+\frac{5}{4} u}+\frac{4}{\left(\sqrt{2}+\frac{5}{4} u\right)^{2}}<2 \frac{2^{-\frac{e}{2}} a+\frac{b}{2}}{a^{2}+b^{2}}+2 \frac{2+2^{-e}}{\left(a^{2}+b^{2}\right)^{2}} \quad \text { for }(a, b, e) \in D_{1} .
\end{aligned}
$$


Since both $\frac{\partial g_{1}}{\partial a}$ and $\frac{\partial g_{1}}{\partial b}$ are negative over $D_{1}$, since $(a, b, e) \in D_{1}$ implies $a \geqslant 2^{-\frac{e}{2}}$ and $b \geqslant 1$, and since $\left(2^{-\frac{e}{2}}, 1, e\right) \in D_{1}$, we deduce that $g_{1}(a, b, e) \leqslant$ $g_{1}\left(2^{-\frac{e}{2}}, 1, e\right)=: h_{1}(x)$, with $x=2^{-e}$ and

$$
h_{1}(x)=(x+1)\left(x+\frac{1}{4}\right)+\frac{(x+2)(2 x+1)}{x+1}+\left(\frac{x+2}{x+1}\right)^{2} .
$$

Since $e \geqslant 2$, we have $0<x \leqslant \frac{1}{4}$ and

$$
h_{1}^{\prime}(x)=\frac{8 x^{4}+37 x^{3}+63 x^{2}+43 x+1}{4(x+1)^{3}}>0 .
$$

Overall, we thus have $f_{2}(a, b) \leqslant g_{1}(a, b, e) \leqslant h_{1}(x) \leqslant h_{1}\left(\frac{1}{4}\right)=6.565$.

3.5 Case $\operatorname{ufp}\left(s_{a}+s_{b}\right)=4$

From (15) and (11), we know that $4 \leqslant s_{a}+s_{b}<4 \sqrt{2}$ and $s_{a}<s_{b}$. As a consequence, we have $2<s_{b}$ which implies $2<b^{2}$, so that

$$
\operatorname{ufp}\left(b^{2}\right)=2 \quad \text { and } \quad \sqrt{2}<b \leqslant 2-2 u .
$$

Since 4 is a floating-point number, we have $s=\operatorname{RN}\left(s_{a}+s_{b}\right) \geqslant 4$ and $\frac{b}{s} \leqslant \frac{b}{4}<$ $\frac{1}{2}$ hence

$$
\mathrm{ufp}\left(\frac{b}{s}\right) \leqslant \frac{1}{4}
$$

In the same way, $\frac{a}{s} \leqslant \frac{a}{4}<2^{-\frac{3+e}{2}}$ so that

$$
\operatorname{ufp}\left(\frac{a}{s}\right) \leqslant 2^{-2-\frac{e}{2}} .
$$

We now distinguish two subcases, namely $e=-1$ and $e \geqslant 0$.

\subsubsection{Subcase $e=-1$}

We have ufp $\left(\frac{a}{s}\right) \leqslant 2^{-\frac{3}{2}}$, hence $\operatorname{ufp}\left(\frac{a}{s}\right) \leqslant \frac{1}{4}$, thus we deduce from (8) that $f_{2}(a, b) \leqslant g_{2}(a, b)$ with

$$
g_{2}(a, b):=\frac{a^{2}+b^{2}}{8}+\frac{4(a+b)}{a^{2}+b^{2}}+\left(\frac{8}{a^{2}+b^{2}}\right)^{2} .
$$

From (15), we know that $s_{a}+s_{b}<4 \sqrt{2}$, which implies $a^{2}+b^{2}<4 \sqrt{2}+4 u$. The domain of interest is then given by

$$
D_{2}:=\left\{(a, b) \mid \sqrt{2} \leqslant a \leqslant b<2, a^{2}+b^{2}<4 \sqrt{2}+4 u\right\} .
$$

Computing the partial derivatives of $g_{2}$ with respect to $a$ and $b$, and proving that they are both negative over the domain $D_{2}$ (detailed computations are in $\S$ A.2), we end up with $f_{2}(a, b) \leqslant g_{2}(\sqrt{2}, \sqrt{2})=\left(2+\frac{\sqrt{2}}{2}\right)^{2}$. 


\subsubsection{Subcase $e \geqslant 0$}

Since $|\delta| \leqslant \operatorname{ufp}\left(a^{2}\right)+\operatorname{ufp}\left(b^{2}\right)+\operatorname{ufp}\left(s_{a}+s_{b}\right)=6+2^{-e}$ and $\operatorname{ufp}\left(\frac{a}{s}\right) \leqslant 2^{-2-\frac{e}{2}}$, from $(8)$ we get $f_{2}(a, b) \leqslant g_{3}(a, b, e)$ with

$$
g_{3}(a, b, e):=\frac{\left(a^{2}+b^{2}\right)\left(2^{-e}+1\right)}{16}+\frac{\left(6+2^{-e}\right)\left(2^{-\frac{e}{2}} a+b\right)}{2\left(a^{2}+b^{2}\right)}+\left(\frac{6+2^{-e}}{a^{2}+b^{2}}\right)^{2} .
$$

From (14), $a^{2}+b^{2}$ is lower bounded by 4 , and we restrict the analysis of $g_{3}$ to the domain

$D_{3}:=\left\{(a, b, e) \mid 2^{-\frac{e}{2}} \leqslant a \leqslant 2^{\frac{1-e}{2}}, \sqrt{2} \leqslant b<2,4 \leqslant a^{2}+b^{2}<4 \sqrt{2}+4 u, e \geqslant 0\right\}$.

First, it can be checked that the partial derivative of $g_{3}$ with respect to $b$ is negative over $D_{3}$ (details are in $\S$ A.3). Since $b \geqslant \sqrt{4-a^{2}}$, and $(a, b, e) \in D_{3}$ implies $\left(a, \sqrt{4-a^{2}}, e\right) \in D_{3}$, we deduce that $g_{3}(a, b, e) \leqslant g_{3}\left(a, \sqrt{4-a^{2}}, e\right)$, where

$$
g_{3}\left(a, \sqrt{4-a^{2}}, e\right)=\frac{2^{-e}+1}{4}+\frac{\left(6+2^{-e}\right)\left(2^{-\frac{e}{2}} a+\sqrt{4-a^{2}}\right)}{8}+\frac{\left(6+2^{-e}\right)^{2}}{16} .
$$

We then compute $\frac{\partial}{\partial a} g_{3}\left(a, \sqrt{4-a^{2}}, e\right)=\frac{6+2^{-e}}{8}\left(2^{-\frac{e}{2}}-\frac{a}{\sqrt{4-a^{2}}}\right)$, which is nonnegative because $a^{2} \leqslant \frac{2 a^{2}}{1+2^{-e}} \leqslant \frac{4 \cdot 2^{-e}}{1+2^{-e}}$. Since $\left(2^{\frac{1-e}{2}}, \sqrt{4-2^{1-e}}, e\right) \in D_{3}$, we have $g_{3}(a, b, e) \leqslant g_{3}\left(2^{\frac{1-e}{2}}, \sqrt{4-2^{1-e}}, e\right)=: h_{3}(x)$, with $x=2^{-e}$ and

$$
h_{3}(x)=\frac{x+1}{4}+\frac{(6+x)(\sqrt{2} x+\sqrt{4-2 x})}{8}+\frac{(6+x)^{2}}{16} .
$$

Since

$$
h_{3}^{\prime}(x)=1+\frac{x}{8}(1+\sqrt{2})+\frac{\sqrt{4-2 x}}{8}+\frac{x+6}{8}\left(\sqrt{2}-\frac{1}{\sqrt{4-2 x}}\right)
$$

is positive for $0<x \leqslant 1$, we deduce $f_{2}(a, b) \leqslant h_{3}(1)=\left(\frac{7}{4}+\frac{\sqrt{2}}{2}\right)^{2}=6.037 \ldots$

3.6 Case ufp $\left(s_{a}+s_{b}\right)=2$

From (14) we have $2 \leqslant a^{2}+b^{2}$, and from (15) we have $2 \leqslant s_{a}+s_{b}<2 \sqrt{2}$ hence

$$
e \geqslant 0 \text {. }
$$

Since 2 is a floating-point number, we know that $s \geqslant 2$. Therefore $\frac{a}{s}<2^{-\frac{1+e}{2}}$, hence

$$
u f p\left(\frac{a}{s}\right) \leqslant 2^{-1-\frac{e}{2}}
$$

and $\frac{b}{s}<1$ so that

$$
u f\left(\frac{b}{s}\right) \leqslant \frac{1}{2}
$$

We handle separately the two possible values, 1 and 2 , for $\operatorname{ufp}\left(b^{2}\right)$. 
3.6.1 Subcase ufp $\left(b^{2}\right)=1$

We distinguish the cases $e \geqslant 1$ and $e=0$.

- Subsubcase $e \geqslant 1$ : From (8) we have $f_{2}(a, b) \leqslant g_{4}(a, b, e)$ with

$$
g_{4}(a, b, e):=\frac{\left(a^{2}+b^{2}\right)\left(2^{-e}+1\right)}{4}+\frac{\left(3+2^{-e}\right)\left(2^{-\frac{e}{2}} a+b\right)}{a^{2}+b^{2}}+\left(\frac{3+2^{-e}}{a^{2}+b^{2}}\right)^{2} .
$$

From (14), we know that $a^{2}+b^{2}$ is lower bounded by 2 . On the other hand, we have $a^{2}+b^{2} \leqslant s_{a}+s_{b}+\left(\operatorname{ufp}\left(a^{2}\right)+\operatorname{ufp}\left(b^{2}\right)\right) u<2 \sqrt{2}+2 u$ and $1<b<\sqrt{2}$, hence we can restrict the analysis to the domain

$D_{4}:=\left\{(a, b, e) \mid 2^{-\frac{e}{2}} \leqslant a<2^{\frac{1-e}{2}}, 1<b<\sqrt{2}, 2 \leqslant a^{2}+b^{2}<2 \sqrt{2}+2 u, e \geqslant 1\right\}$.

We can first compute the partial derivative of $g_{4}$ with respect to $b$ and prove it is negative over $D_{4}$ for $p \geqslant 4$ (see the details in $\S$ A.4). Since $\left(a, \sqrt{2-a^{2}}, e\right.$ ) is in $D_{4}$, we deduce that $g_{4}(a, b, e) \leqslant g_{4}\left(a, \sqrt{2-a^{2}}, e\right)$, and we have

$$
g_{4}\left(a, \sqrt{2-a^{2}}, e\right)=\frac{2^{-e}+1}{2}+\frac{\left(3+2^{-e}\right)\left(2^{-\frac{e}{2}} a+\sqrt{2-a^{2}}\right)}{2}+\frac{\left(3+2^{-e}\right)^{2}}{4} .
$$

Next, we can compute the derivative of $g_{4}\left(a, \sqrt{2-a^{2}}, e\right)$ with respect to $a$ (see $\S$ A.4) and check that the maximum is attained at $a_{0}=2^{-\frac{e}{2}} \sqrt{\frac{2}{1+2^{-e}}}$, so that $g_{4}(a, b, e) \leqslant g_{4}\left(a_{0}, \sqrt{2-a_{0}^{2}}, e\right)=: h_{4}(x)$ with

$$
h_{4}(x)=\frac{x+1}{2}+\frac{3+x}{2}\left(x \sqrt{\frac{2}{1+x}}+\sqrt{2-\frac{2 x}{1+x}}\right)+\frac{(3+x)^{2}}{4} .
$$

Since $h_{4}^{\prime}(x)>0$ for $0<x \leqslant \frac{1}{2}$, we conclude that $f_{2}(a, b) \leqslant g_{4}(a, b, e) \leqslant$ $h_{4}\left(\frac{1}{2}\right)=\left(\frac{7}{4}+\frac{\sqrt{3}}{2}\right)^{2}$.

- Subsubcase $e=0$ : According to (13), we assume that $1<a$, so that $\operatorname{ufp}\left(b^{2}\right)=\operatorname{ufp}\left(a^{2}\right)=1$. It follows that $s \geqslant s_{a}+s_{b}-2 u \geqslant a^{2}+b^{2}-4 u$, hence $\frac{a}{s} \leqslant \frac{a}{a^{2}+b^{2}-4 u}$. Since $a$ and $b$ are both floating-point numbers, and from (11), we know that $b \geqslant a+2 u$ so that $b^{2}-4 u>a^{2}$. By computing its partial derivative, it can then be checked that $\frac{a}{a^{2}+b^{2}-4 u}$ is increasing with respect to $a$, which implies $\frac{a}{s} \leqslant \frac{b-2 u}{(b-2 u)^{2}+b^{2}-4 u}$. This last expression is decreasing with respect to $b$, and since $b \geqslant 1+2 u$ we deduce $\frac{a}{s} \leqslant \frac{1}{2\left(1+2 u^{2}\right)}<\frac{1}{2}$. Thus,

$$
\operatorname{ufp}\left(\frac{a}{s}\right) \leqslant \frac{1}{4}
$$

In the same way, it can be derived from $\frac{b}{s} \leqslant \frac{b}{a^{2}+b^{2}-4 u}$ that

$$
\operatorname{ufp}\left(\frac{b}{s}\right) \leqslant \frac{1}{4}
$$


Combining these bounds on $\operatorname{ufp}\left(\frac{a}{s}\right)$ and $\operatorname{ufp}\left(\frac{b}{s}\right)$ with (8) gives $f_{2}(a, b) \leqslant$ $g_{5}(a, b)$, where

$$
g_{5}(a, b):=\frac{a^{2}+b^{2}}{8}+\frac{2(a+b)}{a^{2}+b^{2}}+\frac{16}{\left(a^{2}+b^{2}\right)^{2}} .
$$

Hence it remains to bound $g_{5}(a, b)$ over the domain $D_{5}$ defined by

$$
D_{5}:=\left\{(a, b) \mid 1 \leqslant a \leqslant b<\sqrt{2} \text { and } a^{2}+b^{2}<2 \sqrt{2}+2 u\right\} .
$$

In this domain, we have $\frac{\partial}{\partial b} g_{5}(a, b)<0$ (details are in $\S$ A.5), so that $g_{5}(a, b) \leqslant$ $g_{5}(a, a)=\frac{a^{2}}{4}+\frac{4}{a^{4}}+\frac{2}{a}$, which is maximal for $a=1$. Therefore, we deduce that $f_{2}(a, b) \leqslant g_{5}(a, b) \leqslant g_{5}(1,1)=\left(\frac{5}{2}\right)^{2}$.

3.6.2 Subcase ufp $\left(b^{2}\right)=2$

In this paragraph, $a^{2}<1$ (otherwise we would have $s_{a}+s_{b} \geqslant 2+1$ while from (15) we have $s_{a}+s_{b}<2 \sqrt{2}$ ), hence $e \geqslant 1$. We split the inequality (16) into two possible cases. Either ufp $\left(\frac{a}{s}\right)<2^{-1-\frac{e}{2}}$ which implies ufp $\left(\frac{a}{s}\right) \leqslant 2^{-\frac{3+e}{2}}$, or $\operatorname{ufp}\left(\frac{a}{s}\right)=2^{-1-\frac{e}{2}}$ in which case $e$ is even.

- Subsubcase ufp $\left(\frac{a}{s}\right)<2^{-1-\frac{e}{2}}$ : We deduce from (8) and $|\delta| \leqslant 4+2^{-e}$ that $f_{2}(a, b) \leqslant g_{6}(a, b, e)$ with

$g_{6}(a, b, e):=\frac{\left(a^{2}+b^{2}\right)\left(2^{-1-e}+1\right)}{4}+\frac{\left(4+2^{-e}\right)\left(2^{-\frac{1+e}{2}} a+b\right)}{a^{2}+b^{2}}+\left(\frac{4+2^{-e}}{a^{2}+b^{2}}\right)^{2}$.

We can compute the derivatives of $g_{6}$ (details are provided in §A.6) with respect to $a$ and $b$ and prove that they are negative over the domain

$$
\begin{aligned}
D_{6}:=\left\{(a, b, e) \mid 2^{-\frac{e}{2}} \leqslant a<2^{\frac{1-e}{2}}, \sqrt{2}\right. & \leqslant b<2, \\
& \left.2 \leqslant a^{2}+b^{2}<2 \sqrt{2}+\left(2+2^{-e}\right) u, e \geqslant 1\right\} .
\end{aligned}
$$

For $(a, b, e) \in D_{6}$, we deduce that $g_{6}(a, b, e) \leqslant g_{6}\left(2^{-\frac{e}{2}}, \sqrt{2}, e\right)=: h_{6}(x)$ with

$$
h_{6}(x)=\frac{(x+2)\left(\frac{x}{2}+1\right)}{4}+\frac{\sqrt{2}(4+x)\left(\frac{x}{2}+1\right)}{x+2}+\left(\frac{4+x}{x+2}\right)^{2}, \quad x=2^{-e} .
$$

We can maximize $h_{6}(x)$ for $0<x \leqslant \frac{1}{2}$, which leads to $f_{2}(a, b) \leqslant h_{6}(0)=$ $\left(2+\frac{\sqrt{2}}{2}\right)^{2}$. 
- Subsubcase ufp $\left(\frac{a}{s}\right)=2^{-1-\frac{e}{2}}$ : In this case, $e$ is even, hence $e \geqslant 2$. We have $f_{2}(a, b) \leqslant g_{7}(a, b, e)$ with

$$
g_{7}(a, b, e):=\frac{\left(a^{2}+b^{2}\right)\left(2^{-e}+1\right)}{4}+\frac{\left(4+2^{-e}\right)\left(2^{-\frac{e}{2}} a+b\right)}{a^{2}+b^{2}}+\left(\frac{4+2^{-e}}{a^{2}+b^{2}}\right)^{2} .
$$

The lower bound $2^{-\frac{e}{2}}$ for $a$ does not lead to a sufficiently tight bound for $f_{2}$ in this case: to get a better bound, we exploit further the hypothesis ufp $\left(\frac{a}{s}\right)=$ $2^{-1-\frac{e}{2}}$. This gives $s 2^{-1-\frac{e}{2}} \leqslant a$, which implies $a^{2}-2^{1+\frac{e}{2}} a+b^{2}+\delta u \leqslant 0$, hence

$$
a \geqslant 2^{\frac{e}{2}}-\sqrt{2^{e}-2+\left(4+2^{-e}\right) u}=a_{0}+\eta(u)
$$

with

$$
a_{0}=2^{\frac{e}{2}}-\sqrt{2^{e}-2}, \quad \eta(u)<0, \quad|\eta(u)| \in \mathcal{O}(u) .
$$

Therefore, we analyze $g_{7}$ over the domain

$$
\begin{aligned}
D_{7}:=\left\{(a, b, e) \mid a_{0}+\eta(u)\right. & \leqslant a<2^{\frac{1-e}{2}}, \sqrt{2} \leqslant b<2, \\
2 & \left.\leqslant a^{2}+b^{2}<2 \sqrt{2}+\left(2+2^{-e}\right) u, e \geqslant 2, e \text { even }\right\} .
\end{aligned}
$$

First, we can compute the partial derivative of $g_{7}$ with respect to $b$ and prove (see Appendix A.7) that it is negative over the domain $D_{7}$, hence we know that $g_{7}(a, b, e) \leqslant g_{7}(a, \sqrt{2}, e)$.

It can be checked that $a_{0}+\eta(u)$ belongs to $\left[2^{-1-\frac{e}{2}}, 2^{\frac{1-e}{2}}\right]$ and that $g_{7}(a, \sqrt{2}, e)$ is decreasing with respect to $a$ over $\left[2^{-1-\frac{e}{2}}, 2^{\frac{1-e}{2}}\right]$; see Appendix A.7. We then deduce that $g_{7}(a, \sqrt{2}, e) \leqslant g_{7}\left(a_{0}+\eta(u), \sqrt{2}, e\right)$, for any $(a, b, e) \in D_{7}$.

Next, it can be proved that $g_{7}\left(a_{0}+\eta(u), \sqrt{2}, e\right) \leqslant g_{7}\left(a_{0}, \sqrt{2}, e\right)+20 u$ (again, the details are provided in $\S$ A.7). As a consequence, for any $(a, b, e) \in D_{7}$ we have $g_{7}(a, b, e) \leqslant g_{7}\left(a_{0}, \sqrt{2}, e\right)+20 u$.

The last step is to bound $g_{7}\left(a_{0}, \sqrt{2}, e\right)$ for $e$ an even positive integer. With $y=\sqrt{1-2^{1-e}}$, we have $g_{7}\left(a_{0}, \sqrt{2}, e\right)=: h_{7}(y)$ with $h_{7}(y)$ a rational function in $y$. The variable $y$ belongs to $[\sqrt{2} / 2,1]$, and $h_{7}^{\prime}(y)=\frac{P(y)}{32(y+1)^{2}}$ with

$$
\begin{aligned}
P(y)=3 y^{7}+11 y^{6}-5 y^{5}- & (12 \sqrt{2}+85) y^{4}-(32 \sqrt{2}+143) y^{3} \\
& -(23-8 \sqrt{2}) y^{2}+(64 \sqrt{2}+113) y+36 \sqrt{2}+33 .
\end{aligned}
$$

Using Descartes' rule of signs, one can check that $P$ has exactly one root in the interval $[\sqrt{2} / 2,1]$, and since the evaluation of $P$ is positive at $\sqrt{1-2^{-5}}$ and negative at $\sqrt{1-2^{-7}}$, we deduce that $h_{7}$ is increasing over $\left[\sqrt{2} / 2, \sqrt{1-2^{-5}}\right]$ and decreasing over $\left[\sqrt{1-2^{-7}}, 1\right]$. Comparing the values of $h_{7}$ at the points $\sqrt{1-2^{-5}}$ and $\sqrt{1-2^{-7}}$, we conclude that $h_{7}\left(\sqrt{1-2^{-7}}\right)$ is an upper bound for $h_{7}$.

Finally, it can be checked that $h_{7}\left(\sqrt{1-2^{-7}}\right)=\gamma^{2}$ hence we get $f_{2}(a, b) \leqslant$ $\gamma^{2}+20 u$. From (9), we derive the final upper bound $\gamma u+9 u^{2}$ for $\mathrm{E}_{\mathrm{N}}$, which concludes the proof of Theorem 2 . 


\section{Implications for complex floating-point division}

Let us conclude with some remarks about complex division. The conventional complex division algorithm for computing an approximation $\widehat{z}=\widehat{R}+i \widehat{I}$ of $(a+i b) /(c+i d)$ in floating-point arithmetic consists in evaluating the real part as

$$
\widehat{R}=\operatorname{RN}\left(\frac{\operatorname{RN}(\operatorname{RN}(a c)+\mathrm{RN}(b d))}{\operatorname{RN}\left(\operatorname{RN}\left(c^{2}\right)+\operatorname{RN}\left(d^{2}\right)\right)}\right)
$$

and using a similar scheme for the imaginary part. An approximate quotient $\widehat{z}$ can also be obtained by first computing an inverse of $c+i d$ using Algorithm 1 , and then multiplying it by $a+i b$ by means of the classic complex multiplication algorithm. Note that both algorithms require 3 additions/subtractions, 6 multiplications, and 2 divisions.

Normwise relative accuracy analyses of the method based on (17) can be found in $[4,12,5]$. To our knowledge, the best known upper bound for the normwise relative error generated by this method is $(3+\sqrt{5}) u+\mathcal{O}\left(u^{2}\right) \approx 5.2 u$ : as noted in [1], this bound can be derived from the bound $\sqrt{5} u$ from [3] on the normwise relative error for the classic complex multiplication algorithm. On the other hand, it can be checked using Theorem 2 and, again, the bound $\sqrt{5} u$ from $[3]$ that the algorithm combining inversion and multiplication admits the smaller normwise error bound $(\gamma+\sqrt{5}) u+\mathcal{O}\left(u^{2}\right) \approx 4.9 u$. The following examples of complex quotients in precision $p=11$ show that in both cases the largest normwise relative error cannot be bounded by $\gamma u+\mathcal{O}\left(u^{2}\right) \approx 2.7 u$ as for inversion:

- with $a+i b=1575+i 1419$ and $c+i d=1457+i 1480$, using (17) gives $|\widehat{z}-z| /(u|z|)=4.67973 \ldots$;

- dividing $1506+i 1512$ by $1491+i 1504$ using the inversion-multiplication approach leads to $|\widehat{z}-z| /(u|z|)=4.34446 \ldots$

However, these examples are not sufficient to conclude about the sharpness of the bounds $(3+\sqrt{5}) u+\mathcal{O}\left(u^{2}\right)$ and $(\gamma+\sqrt{5}) u+\mathcal{O}\left(u^{2}\right)$, and further investigation is needed to understand the accuracy of complex floating-point division.

\section{Acknowledgements}

We thank the associate editor and the anonymous reviewers for their helpful comments and suggestions.

\section{References}

1. Baudin, M.: Error bounds of complex arithmetic (2011). Available at http://forge. scilab.org/upload/compdiv/files/complexerrorbounds_v0.2.pdf

2. Baudin, M., Smith, R.L.: A robust complex division in Scilab (2012). Available at http://arxiv.org/abs/1210.4539 
3. Brent, R., Percival, C., Zimmermann, P.: Error bounds on complex floating-point multiplication. Mathematics of Computation 76, 1469-1481 (2007)

4. Champagne, W.P.: On finding roots of polynomials by hook or by crook. Master's thesis, University of Texas (1964)

5. Higham, N.J.: Accuracy and Stability of Numerical Algorithms, second edn. SIAM, Philadelphia, PA, USA (2002)

6. IEEE Computer Society: IEEE Standard for Floating-Point Arithmetic. IEEE Standard 754-2008 (2008). Available at http://ieeexplore.ieee.org/servlet/opac?punumber= 4610933

7. Knuth, D.E.: The Art of Computer Programming, Volume 2, Seminumerical Algorithms, third edn. Addison-Wesley, Reading, MA, USA (1998)

8. Priest, D.M.: Efficient scaling for complex division. ACM Transactions on Mathematical Software 30(4) (2004)

9. Rump, S.M., Ogita, T., Oishi, S.: Accurate floating-point summation, Part I: Faithful rounding. SIAM Journal on Scientific Computing 31(1), 189-224 (2008)

10. Smith, R.L.: Algorithm 116: Complex division. Communications of the ACM 5(8), 435 (1962)

11. Stewart, G.W.: A note on complex division. ACM Transactions on Mathematical Software 11(3), 238-241 (1985)

12. Wilkinson, J.H.: The Algebraic Eigenvalue Problem. Oxford University Press (1965)

13. Ziv, A.: Sharp ULP rounding error bound for the hypotenuse function. Mathematics of Computation 68(227), 1143-1148 (1999) 


\section{A Details omitted in the proofs}

A.1 Asymptotic optimality of the componentwise error bound

We briefly detail the computations of $s_{a}, s_{b}$ and $s$ in the example parametrized by $p$ given in Section 2. We assume that $p \geqslant 12$ is even, and we recall that

$$
\begin{aligned}
& a=2^{\frac{p}{2}-1}+5 \cdot 2^{-2}+2^{-\frac{p}{2}+2}, \\
& b=2^{p-1}+2^{\frac{p}{2}-1}+1 .
\end{aligned}
$$

- Computation of $s_{a}=\mathrm{RN}\left(a^{2}\right)$ :

$$
\begin{aligned}
a^{2} & =2^{p-2}+5 \cdot 2^{\frac{p}{2}-2}+11 \cdot 2^{-1}+2^{-4}+10 \cdot 2^{-\frac{p}{2}}+2^{-p+4} \\
\operatorname{ulp}\left(a^{2}\right) & =2^{-1} \\
\widetilde{s_{a}} & :=2^{p-2}+5 \cdot 2^{\frac{p}{2}-2}+11 \cdot 2^{-1} \\
\left|a^{2}-\widetilde{s_{a}}\right| & =2^{-4}+10 \cdot 2^{-\frac{p}{2}}+2^{4-p} \\
& \leqslant 2^{-4}+10 \cdot 2^{-6}+2^{-8} \\
& <2^{-2}=\frac{1}{2} \operatorname{ulp}\left(a^{2}\right)
\end{aligned}
$$

Hence $s_{a}=\widetilde{s_{a}}$.

- Computation of $s_{b}=\mathrm{RN}\left(b^{2}\right)$ :

$$
\begin{aligned}
b^{2} & =2^{2 p-2}+2^{\frac{3 p}{2}-1}+2^{p}+2^{p-2}+2^{\frac{p}{2}}+1 \\
\widetilde{s_{b}} & :=2^{2 p-2}+2^{\frac{3 p}{2}-1}+3 \cdot 2^{p-1} \\
\operatorname{ulp}\left(b^{2}\right) & =2^{p-1} \\
\left|b^{2}-\widetilde{s_{b}}\right| & =2^{p-2}-2^{\frac{p}{2}}-1 \\
& <2^{p-2}=\frac{1}{2} \mathrm{ulp}\left(b^{2}\right)
\end{aligned}
$$

Hence $s_{b}=\widetilde{s_{b}}$.

- Computation of $s=\mathrm{RN}\left(s_{a}+s_{b}\right)$

$$
\begin{aligned}
s_{a}+s_{b} & =2^{2 p-2}+2^{\frac{3 p}{2}-1}+3 \cdot 2^{p-1}+2^{p-2}+5 \cdot 2^{\frac{p}{2}-2}+11 \cdot 2^{-1} \\
\widetilde{s} & =2^{2 p-2}+2^{\frac{3 p}{2}-1}+2^{p+1} \\
\operatorname{ulp}\left(s_{a}+s_{b}\right) & =2^{p-1} \\
\left|s_{a}+s_{b}-\widetilde{s}\right| & =2^{p-2}-5 \cdot 2^{\frac{p}{2}-2}-11 \cdot 2^{-1} \\
& <2^{p-2}=\frac{1}{2} \operatorname{ulp}\left(s_{a}+s_{b}\right)
\end{aligned}
$$

Hence $s=\widetilde{s}$ 
A.2 Partial derivatives of $g_{2}$

Computing the partial derivatives of $g_{2}$ with respect to $a$ and $b$ gives

$$
\begin{aligned}
& \frac{\partial g_{2}}{\partial a}=\frac{a}{4}+\frac{4}{a^{2}+b^{2}}-\frac{8 a(a+b)}{\left(a^{2}+b^{2}\right)^{2}}-\frac{256 a}{\left(a^{2}+b^{2}\right)^{3}}, \\
& \frac{\partial g_{2}}{\partial b}=\frac{b}{4}+\frac{4}{a^{2}+b^{2}}-\frac{8 b(a+b)}{\left(a^{2}+b^{2}\right)^{2}}-\frac{256 b}{\left(a^{2}+b^{2}\right)^{3}} .
\end{aligned}
$$

First, we know that $b>a$ so $\frac{1}{b} \frac{\partial}{\partial b} g_{2}(a, b)<\frac{1}{a} \frac{\partial}{\partial a} g_{2}(a, b)$. We just have to prove that $\frac{\partial}{\partial a} g_{2}(a, b)<0$, that is,

$$
\frac{\left(a^{2}+b^{2}\right)^{2}}{4}+\frac{4\left(a^{2}+b^{2}\right)}{a}<8(a+b)+\frac{256}{a^{2}+b^{2}} .
$$

Since for $(a, b) \in D_{2}$ we have $\sqrt{2}<a, b$, and $a^{2}+b^{2}<4 \sqrt{2}+4 u$, it is enough to check that

$$
\frac{(4 \sqrt{2}+4 u)^{2}}{4}+\frac{4(4 \sqrt{2}+4 u)}{\sqrt{2}}<16 \sqrt{2}+\frac{256}{4 \sqrt{2}+4 u},
$$

which holds for $p \geqslant 2$.

\section{A.3 Partial derivative of $g_{3}$}

We compute the partial derivative of $g_{3}$ with respect to $b$, and check that this derivative is negative over the domain $D_{3}$. We have

$$
\frac{\partial g_{3}}{\partial b}=\frac{b\left(2^{-e}+1\right)}{8}+\frac{6+2^{-e}}{2\left(a^{2}+b^{2}\right)}-b \frac{\left(6+2^{-e}\right)\left(2^{-\frac{e}{2}} a+b\right)}{\left(a^{2}+b^{2}\right)^{2}}-4 b \frac{\left(6+2^{-e}\right)^{2}}{\left(a^{2}+b^{2}\right)^{3}},
$$

and we check that

$$
\frac{b\left(2^{-e}+1\right)}{8}+\frac{6+2^{-e}}{2\left(a^{2}+b^{2}\right)}<b \frac{\left(6+2^{-e}\right)\left(2^{-\frac{e}{2}} a+b\right)}{\left(a^{2}+b^{2}\right)^{2}}+4 b \frac{\left(6+2^{-e}\right)^{2}}{\left(a^{2}+b^{2}\right)^{3}} .
$$

Multiplying both sides by $\frac{\left(a^{2}+b^{2}\right)^{2}}{b\left(6+2^{-e}\right)}$ and since $1 \leqslant b$, it is enough to prove that

$$
\frac{\left(2^{-e}+1\right)\left(a^{2}+b^{2}\right)^{2}}{8\left(6+2^{-e}\right)}+\frac{a^{2}+b^{2}}{2}<2^{-\frac{e}{2}} a+b+4 \frac{6+2^{-e}}{a^{2}+b^{2}} .
$$

This follows from the following sequence of three inequalities

$$
\begin{aligned}
\frac{\left(2^{-e}+1\right)\left(a^{2}+b^{2}\right)^{2}}{8\left(6+2^{-e}\right)}+\frac{a^{2}+b^{2}}{2} & <\frac{2(4 \sqrt{2}+4 u)^{2}}{48}+\frac{4 \sqrt{2}+4 u}{2} \\
\frac{2(4 \sqrt{2}+4 u)^{2}}{48}+\frac{4 \sqrt{2}+4 u}{2} & <4 \frac{6}{4 \sqrt{2}+4 u}+1 \quad \text { for } p \geqslant 3 \\
4 \frac{6}{4 \sqrt{2}+4 u}+1 & <4 \frac{6+2^{-e}}{a^{2}+b^{2}}+2^{-\frac{e}{2}} a+b
\end{aligned}
$$


A.4 Partial derivatives of $g_{4}$

The partial derivative of $g_{4}$ with respect to $b$ is given by

$$
\frac{\partial g_{4}}{\partial b}=\frac{b\left(2^{-e}+1\right)}{2}+\frac{3+2^{-e}}{a^{2}+b^{2}}-2 b \frac{\left(2^{-\frac{e}{2}} a+b\right)\left(3+2^{-e}\right)}{\left(a^{2}+b^{2}\right)^{2}}-4 b \frac{\left(3+2^{-e}\right)^{2}}{\left(a^{2}+b^{2}\right)^{3}} .
$$

We want to prove that $\frac{\partial}{\partial b} g_{4}(a, b, e)<0$ or, equivalently, that

$$
\frac{\left(a^{2}+b^{2}\right)^{2}\left(2^{-e}+1\right)}{2\left(3+2^{-e}\right)}+\frac{a^{2}+b^{2}}{b}<2\left(2^{-\frac{e}{2}} a+b\right)+4 \frac{3+2^{-e}}{a^{2}+b^{2}} .
$$

This inequality can be derived from the following ones:

$$
\begin{aligned}
\frac{\left(a^{2}+b^{2}\right)^{2}\left(2^{-e}+1\right)}{2\left(3+2^{-e}\right)}+\frac{a^{2}+b^{2}}{b} & <\frac{2(2 \sqrt{2}+2 u)^{2}}{6}+2 \sqrt{2}+2 u, \\
\frac{(2 \sqrt{2}+2 u)^{2}}{3}+2 \sqrt{2}+2 u & <2+\frac{12}{2 \sqrt{2}+2 u} \quad \text { for } p \geqslant 4, \\
2+\frac{12}{2 \sqrt{2}+2 u} & <2\left(2^{\left.-\frac{e}{2} a+b\right)+4 \frac{3+2^{-e}}{a^{2}+b^{2}}} .\right.
\end{aligned}
$$

The partial derivative of $g_{4}\left(a, \sqrt{2-a^{2}}, e\right)$ with respect to $a$ is

$$
\frac{\partial}{\partial a} g_{4}\left(a, \sqrt{2-a^{2}}, e\right)=\frac{3+2^{-e}}{2}\left(2^{-\frac{e}{2}}-\frac{a}{\sqrt{2-a^{2}}}\right),
$$

which is zero if $a=a_{0}$ with $a_{0}=2^{-\frac{e}{2}} \sqrt{\frac{2}{1+2^{-e}}}$, positive if $a<a_{0}$, and negative if $a>a_{0}$.

\section{A.5 Partial derivative of $g_{5}$}

We have

$$
\frac{\partial g_{5}}{\partial b}=\frac{b}{4}+\frac{2}{a^{2}+b^{2}}-\frac{4(a+b)}{\left(a^{2}+b^{2}\right)^{2}} b-\frac{64}{\left(a^{2}+b^{2}\right)^{3}} b
$$

and it can be checked that this partial derivative is negative using the following inequalities:

$$
\begin{gathered}
\frac{\left(a^{2}+b^{2}\right)^{2}}{4}+\frac{2}{b}\left(a^{2}+b^{2}\right)<\frac{(2 \sqrt{2}+2 u)^{2}}{4}+2(2 \sqrt{2}+2 u), \\
\frac{(2 \sqrt{2}+2 u)^{2}}{4}+2(2 \sqrt{2}+2 u)<8+\frac{64}{2 \sqrt{2}+2 u} \quad \text { for } p \geqslant 2, \\
8+\frac{64}{2 \sqrt{2}+2 u}<4(a+b)+\frac{64}{a^{2}+b^{2}} .
\end{gathered}
$$

A.6 Partial derivatives of $g_{6}$

The partial derivatives of $g_{6}$ with respect to $a$ and $b$ are

$$
\frac{\partial g_{6}}{\partial a}=\frac{a}{4}\left(2^{-e}+2\right)+\frac{4+2^{-e}}{a^{2}+b^{2}} 2^{-\frac{1+e}{2}}-2 a \frac{\left(2^{-\frac{1+e}{2}} a+b\right)\left(4+2^{-e}\right)}{\left(a^{2}+b^{2}\right)^{2}}-4 a \frac{\left(4+2^{-e}\right)^{2}}{\left(a^{2}+b^{2}\right)^{3}}
$$


and

$$
\frac{\partial g_{6}}{\partial b}=\frac{b}{4}\left(2^{-e}+2\right)+\frac{4+2^{-e}}{a^{2}+b^{2}}-2 b \frac{\left(2^{-\frac{1+e}{2}} a+b\right)\left(4+2^{-e}\right)}{\left(a^{2}+b^{2}\right)^{2}}-4 b \frac{\left(4+2^{-e}\right)^{2}}{\left(a^{2}+b^{2}\right)^{3}} .
$$

For $(a, b, e) \in D_{6}$, it can be checked that $\frac{\partial g_{6}}{\partial a}(a, b, e)<0$ and $\frac{\partial g_{6}}{\partial b}(a, b, e)<0$ as follows. Note first that $2^{-\frac{e}{2}} \leqslant a$ implies

$$
\frac{4+2^{-e}}{a^{2}+b^{2}} 2^{-\frac{1+e}{2}} \leqslant \frac{4+2^{-e}}{\sqrt{2}\left(a^{2}+b^{2}\right)} a,
$$

and that $\sqrt{2} \leqslant b$ implies

$$
\frac{4+2^{-e}}{a^{2}+b^{2}} \leqslant \frac{4+2^{-e}}{\sqrt{2}\left(a^{2}+b^{2}\right)} b .
$$

Thus, the same expression can be used as an upper bound for both $\frac{1}{a} \frac{\partial g_{6}}{\partial a}$ and $\frac{1}{b} \frac{\partial g_{6}}{\partial b}$. Then, multiplying it by $\frac{\left(a^{2}+b^{2}\right)^{2}}{4+2^{-e}}$, it is enough to prove that

$$
\frac{\left(a^{2}+b^{2}\right)^{2}\left(2^{-1-e}+1\right)}{2\left(4+2^{-e}\right)}+\frac{a^{2}+b^{2}}{\sqrt{2}}<2\left(2^{-\frac{1+e}{2}} a+b\right)+4 \frac{4+2^{-e}}{a^{2}+b^{2}} .
$$

This last inequality follows from the following three ones:

$$
\begin{aligned}
& \frac{\left(a^{2}+b^{2}\right)^{2}\left(2^{-1-e}+1\right)}{2\left(4+2^{-e}\right)}+\frac{a^{2}+b^{2}}{\sqrt{2}}<\frac{\left(2 \sqrt{2}+\left(2+\frac{1}{2}\right) u\right)^{2}\left(\frac{1}{4}+1\right)}{8}+2+\frac{2+\frac{1}{2}}{\sqrt{2}} u, \\
& \frac{\left(2 \sqrt{2}+\left(2+\frac{1}{2}\right) u\right)^{2}\left(\frac{1}{4}+1\right)}{8}+2+\frac{2+\frac{1}{2}}{\sqrt{2}} u<2 \sqrt{2}+\frac{16}{2 \sqrt{2}+\left(2+\frac{1}{2}\right) u} \quad \text { for } p \geqslant 2,
\end{aligned}
$$

and

$$
2 \sqrt{2}+\frac{16}{2 \sqrt{2}+\left(2+\frac{1}{2}\right) u}<2\left(2^{-\frac{1+e}{2}} a+b\right)+4 \frac{4+2^{-e}}{a^{2}+b^{2}} .
$$

A.7 Analysis of $g_{7}$

In this section, we provide some details about the analysis of $g_{7}$ that were omitted in §3.6.2.

- Let us first maximize $g_{7}$ with respect to $b$. We have

$$
\frac{\partial g_{7}}{\partial b}=\frac{b}{2}\left(2^{-e}+1\right)+\frac{4+2^{-e}}{a^{2}+b^{2}}-2 b \frac{\left(2^{-\frac{e}{2}} a+b\right)\left(4+2^{-e}\right)}{\left(a^{2}+b^{2}\right)^{2}}-4 b \frac{\left(4+2^{-e}\right)^{2}}{\left(a^{2}+b^{2}\right)^{3}} .
$$

We want to prove that $\frac{\partial}{\partial b} g_{7}(a, b, e)<0$ over $D_{7}$. Multiplying by $\frac{\left(a^{2}+b^{2}\right)^{2}}{\left(4+2^{-e}\right) b}$ and using the inequality $\frac{1}{b}<1$, we only need to prove that

$$
\frac{\left(a^{2}+b^{2}\right)^{2}\left(2^{-e}+1\right)}{2\left(4+2^{-e}\right)}+a^{2}+b^{2}<2\left(2^{-\frac{e}{2}} a+b\right)+4 \frac{4+2^{-e}}{a^{2}+b^{2}} .
$$

Since $e \geqslant 2$, we can derive this inequality for $p \geqslant 2$ from the three following ones using the definition of $D_{7}$ :

$$
\frac{\left(a^{2}+b^{2}\right)^{2}\left(2^{-e}+1\right)}{2\left(4+2^{-e}\right)}+a^{2}+b^{2}<\frac{\left(2 \sqrt{2}+\left(2+\frac{1}{4}\right) u\right)^{2}\left(\frac{1}{4}+1\right)}{8}+2 \sqrt{2}+\left(2+\frac{1}{4}\right) u,
$$




$$
\frac{\left(2 \sqrt{2}+\left(2+\frac{1}{4}\right) u\right)^{2}\left(\frac{1}{4}+1\right)}{8}+2 \sqrt{2}+\left(2+\frac{1}{4}\right) u<2 \sqrt{2}+\frac{16}{2 \sqrt{2}+\left(2+\frac{1}{4}\right) u}
$$

and

$$
2 \sqrt{2}+\frac{16}{2 \sqrt{2}+\left(2+\frac{1}{4}\right) u}<2\left(2^{-\frac{e}{2}} a+b\right)+4 \frac{4+2^{-e}}{a^{2}+b^{2}} .
$$

Therefore, $g_{7}$ is decreasing with respect to $b$, and for all $(a, b, e)$ in $D_{7}, g_{7}(a, b, e) \leqslant g_{7}(a, \sqrt{2}, e)$.

- We now maximize $g_{7}(a, \sqrt{2}, e)$ with respect to $a$. Let us recall that in $D_{7}$,

$$
a \geqslant a_{0}+\eta(u)=2^{\frac{e}{2}}-\sqrt{2^{e}-2+\left(4+2^{-e}\right) u}
$$

and prove that

$$
a_{0}+\eta(u) \geqslant 2^{-1-\frac{e}{2}} .
$$

Using the notation $x=2^{-e}$, the inequality $a_{0}+\eta(u) \geqslant 2^{-1-\frac{e}{2}}$ is equivalent to $\left(\frac{1}{4}-u\right) x \geqslant$ $-1+4 u$ which holds for $p \geqslant 2$ since $\frac{1}{4}-u \geqslant 0 \geqslant-1+4 u$.

Moreover, we have

$$
\begin{array}{r}
\frac{\left(a^{2}+2\right)^{2}}{a\left(4+2^{-e}\right)} \frac{\partial}{\partial a} g_{7}(a, \sqrt{2}, e)=\frac{\left(2^{-e}+1\right)\left(a^{2}+2\right)^{2}}{2\left(4+2^{-e}\right)}+\frac{2^{-\frac{e}{2}}}{a}\left(a^{2}+2\right) \\
-2\left(2^{-\frac{e}{2}} a+\sqrt{2}\right)-4 \frac{4+2^{-e}}{a^{2}+2}
\end{array}
$$

with $\frac{\left(a^{2}+2\right)^{2}}{a\left(4+2^{-e}\right)}>0$ for $a \in I:=\left[2^{-1-\frac{e}{2}}, 2^{\frac{1-e}{2}}\right]$. For $e \geqslant 2$ and $a \in I$, we have

$$
\frac{\left(a^{2}+2\right)^{2}}{a\left(4+2^{-e}\right)} \frac{\partial}{\partial a} g_{7}(a, \sqrt{2}, e)<\frac{125}{128}+5-2 \sqrt{2}-\frac{32}{5}<0 .
$$

As a consequence, $g_{7}(a, \sqrt{2}, e)$ is decreasing with respect to $a$ over $I$, and since $a_{0}+\eta(u) \in I$, the maximum of $g_{7}(a, \sqrt{2}, e)$ for $a \in\left[a_{0}+\eta(u), 2^{\frac{1-e}{2}}\right]$ is $g_{7}\left(a_{0}+\eta(u), \sqrt{2}, e\right)$.

Thus, for $(a, b, e)$ in $D_{7}$, we have $g_{7}(a, b, e) \leqslant g_{7}(a, \sqrt{2}, e) \leqslant g_{7}\left(a_{0}+\eta(u), \sqrt{2}, e\right)$.

- Let us prove that $g_{7}\left(a_{0}+\eta(u), \sqrt{2}, e\right) \leqslant g_{7}\left(a_{0}, \sqrt{2}, e\right)+20 u$. For this purpose, we first show that $|\eta(u)|<2 u$. We have

$$
|\eta(u)|=\sqrt{2^{e}-2+\left(4+2^{-e}\right) u}-\sqrt{2^{e}-2}
$$

and a short calculation shows that $|\eta(u)|<2 u$. It can also be checked that $a_{0}<2^{\frac{1-e}{2}}$ using again $x=2^{-e}$ and a short calculation. Since $e \geqslant 2$, this implies $a_{0} \leqslant \frac{\sqrt{2}}{2}<1$. Let us now consider

$$
\lambda_{0}(u)=\frac{1}{\left(a_{0}+\eta(u)\right)^{2}+2} .
$$

We have

$$
\lambda_{0}(u)=\frac{1}{a_{0}^{2}+2}-\frac{2 a_{0}+\eta(u)}{\left(a_{0}^{2}+2\right)\left(\left(a_{0}+\eta(u)\right)^{2}+2\right)} \eta(u),
$$

and using $-2 u<\eta(u) \leqslant 0$, we deduce

$$
\lambda_{0}(u)<\frac{1}{a_{0}^{2}+2}+a_{0} u
$$


Moreover, we have

$$
\begin{aligned}
\lambda_{0}(u)^{2}=\left(\frac{1}{a_{0}^{2}+2}\right)^{2}-\frac{4 a_{0}}{\left(a_{0}^{2}+2\right)^{2}\left(\left(a_{0}+\right.\right.} & \left.\eta(u))^{2}+2\right) \\
& \\
& +\frac{\left(2 a_{0}+\eta(u)\right)^{2}-2\left(\left(a_{0}+\eta(u)\right)^{2}+2\right)}{\left(a_{0}^{2}+2\right)^{2}\left(\left(a_{0}+\eta(u)\right)^{2}+2\right)^{2}} \eta(u)^{2}
\end{aligned}
$$

and using both $-2 u<\eta(u) \leqslant 0$ and $a_{0}<1$, we also deduce

$$
\lambda_{0}(u)^{2}<\left(\frac{1}{a_{0}^{2}+2}\right)^{2}+a_{0} u
$$

From the definition of $g_{7}$, using $0<a_{0}+\eta(u)<a_{0}$ and the previous upper bounds on $\lambda_{0}(u)$ and $\lambda_{0}(u)^{2}$, we obtain

$$
\begin{array}{r}
g_{7}\left(a_{0}+\eta(u), \sqrt{2}, e\right)<\frac{\left(a_{0}^{2}+2\right)\left(2^{-e}+1\right)}{4}+\left(4+2^{-e}\right)\left(2^{-\frac{e}{2}} a_{0}+\sqrt{2}\right)\left(\frac{1}{a_{0}^{2}+2}+a_{0} u\right) \\
+\left(4+2^{-e}\right)^{2}\left(\frac{1}{\left(a_{0}^{2}+2\right)^{2}}+a_{0} u\right)
\end{array}
$$

and $g_{7}\left(a_{0}+\eta(u), \sqrt{2}, e\right)<g_{7}\left(a_{0}, \sqrt{2}, e\right)+\left(4+2^{-e}\right)\left(2^{-\frac{e}{2}} a_{0}+\sqrt{2}+4+2^{-e}\right) a_{0} u$. The inequality $g_{7}\left(a_{0}+\eta(u), \sqrt{2}, e\right)<g_{7}\left(a_{0}, \sqrt{2}, e\right)+20 u$ then follows from $e \geqslant 2$ and $a_{0} \leqslant \frac{\sqrt{2}}{2}$.

- Finally, we check that the function $h_{7}$ is increasing over $\left[\sqrt{2} / 2, \sqrt{1-2^{-5}}\right]$ and decreasing over $\left[\sqrt{1-2^{-7}}, 1\right]$. We have $h_{7}(y)=\frac{H(y)}{64(y+1)}$ with

$$
\begin{aligned}
H(y)=y^{7}+3 y^{6}-7 y^{5}-(8 \sqrt{2}+ & 45) y^{4}-(16 \sqrt{2}+53) y^{3} \\
& +(64 \sqrt{2}+113) y^{2}+(144 \sqrt{2}+315) y+72 \sqrt{2}+249 .
\end{aligned}
$$

Hence $h_{7}^{\prime}(y)=\frac{P(y)}{32(y+1)^{2}}$ where $P$ is the polynomial

$$
\begin{array}{r}
P(y)=3 y^{7}+11 y^{6}-5 y^{5}-(12 \sqrt{2}+85) y^{4}-(32 \sqrt{2}+143) y^{3} \\
-(23-8 \sqrt{2}) y^{2}+(64 \sqrt{2}+113) y+36 \sqrt{2}+33 .
\end{array}
$$

This polynomial has 0 or 2 positive roots according to Descartes' rule of signs (there are two sign changes in the sequence of coefficients). Moreover,

$$
\begin{aligned}
P(y+1)=3 y^{7}+32 y^{6}+124 y^{5}+ & (160-12 \sqrt{2}) y^{4}-(208+80 \sqrt{2}) y^{3} \\
& -(784+160 \sqrt{2}) y^{2}-(640+64 \sqrt{2}) y-96+64 \sqrt{2},
\end{aligned}
$$

with only one sign change, so there is exactly one root of $P$ greater than 1 and at most one root of $P$ in $[\sqrt{2} / 2,1]$. Since $P\left(\sqrt{1-2^{-5}}\right)>0$ and $P\left(\sqrt{1-2^{-7}}\right)<0$, we deduce that $P(y)$ is positive for $y \in\left[\sqrt{2} / 2, \sqrt{1-2^{-5}}\right]$, and negative for $y \in\left[\sqrt{1-2^{-7}}, 1\right]$, which implies that $h_{7}$ is increasing over the former interval, and decreasing over the latter. 